\title{
Synthesis of Chitin Nano Particles for Preparation of Nano PF Composites
}

\author{
R. S. Umamaheshwar Rao ${ }^{1}$, Dr. G. Chandra Mohan Reddy ${ }^{2}$ \\ ${ }^{1}$ Asst. Professor, MREC, D.No.20-304, Dinakar Nagar Clny, West Venkatapuram, Secunderabad-15 \\ ${ }^{2}$ Professor of Mechanical Engineering \& Principal, MGIT, Gandipet, Hyderabad-75
}

\begin{abstract}
Preparation of Phenolic nanocomposites with the help of solvent casting technique was carried out by dispersing the chitin nano particles in water soluble phenol formaldehyde $(P F)$. The nanocomposites were prepared by using water suspended chitin whisker as the reinforced phase and water soluble phenol formaldehyde (PF) as matrix. In the present work, crab shell originated chitin whisker was processed by acid hydrolysis method to prepare the chitin whiskers. The whiskers suspension consists of parallelepiped slender rod shape which can be used for mixing it with any water soluble thermosetting cross linking polymer. The suspension can be mixed with the water soluble aqueous solution of PF so that the suspension quantify for the varying fraction of chitin whisker particles ranging from 1 to 20 percent. Thus the varying fraction, whisker chitin reinforced phenol formaldehyde composites can be prepared by solvent casting process.
\end{abstract}

Keywords: Chitin Whisker Suspension, Acid Hydrolysis, Water Soluble Aqueous Polymer, Phenolic nano composites.

\section{Introduction}

Polymer composites are developed specially because of the properties such as lightweight, biodegradable, availability, superior mechanical properties, thermal properties, sorption properties etc.. Use of natural filler has been in the process of development of variety of composites for different applications. Researchers have been using these natural fillers as a reinforcing material in preparing composite materials. However, incorporation of these natural fillers do enhance the properties of the matrix material but these fillers if incorporated in composites at a nano scale level the properties will be enhanced further more even for a very little volume or weight fraction. Very few publications can be found based on the phenolics as a matrix material for preparing the composites due to its complicated structure and their technologies and applications, several aspects of their chemistry are still only partially understood. Xiao-ming Tan et.al [1] reported that much work has not been done on the water-developable photocrosslinking polymer except several patents. A limited work has been reported on phenol formaldehyde as a matrix. The use of water soluble phenol formaldehyde was never reported in the past.

The properties of phenol formaldehyde matrix material can be altered by the incorporation of nano scale chitin particles. Preparation of chitin nano particles has been previously reported $[1,2]$ with various matrix. The effect of chitin on mechanical properties was found to be enhanced. Many other properties were also enhanced due to the reinforcement of chitin nano particles. Very limited work has been reported on the synthesis of bio-nanocomposites [2] and no details related to size analysis been reported in the past. Most of the reports have been reported on the use of chitin particle reinforcement as a drug delivery agent [3].

Preliminary studies were performed with chitin whisker obtained from Squid pen and Riftia tubes [4] in the past. In this study the Chitin whisker suspension were prepared by acid hydrolysis method [5, 6] from chitin micro particles obtained from Merine Chemicals Limited, Cochin, Kerala. The source of these chitin particles are from crab shells. The prepared nano fillers will be used to reinforce the water soluble phenol formaldehyde resin obtained from Rishab chemicals limited. Ultrasonic stirring was carried out for uniform distribution of nano particles in the suspension.

\section{Experimental Work}

2.1. Preparation of Chitin Nano Particles: Nano particles have been prepared by acid hydrolysis chemical process. Which includes three basic steps

(i) Decalcification or Bleaching: This involves cleaning by removing any calcium salts present (decalcification) in chitin particles obtained from the crab shells as a main source.

In this the chitin particles are first boiled and stirred simultaneously in 5\% $\mathrm{KOH}$ solution for 6hrs. The sample was subsequently kept at room temperature overnight under stirring. They were further filtered by vacuum pump and washed several times with distilled water until ph 4.

(ii) Deproteinization: In this step the chitin particles are processed to make them protein free. 
The chitin particle suspension which was kept in $5 \% \mathrm{KOH}$ solution for $48 \mathrm{hrs}$ was washed thoroughly until $\mathrm{PH} 4$ to make it a neutral suspension. Chitin samples were then bleached with $17 \mathrm{~g}$ of $\mathrm{NaClO}_{2}$ in $1 \mathrm{~L}$ of water containing $0.3 \mathrm{M}$ sodium acetate buffer, for $6 \mathrm{hrs}$ at $80^{\circ} \mathrm{C}$. The bleaching solution was changed for every $2 \mathrm{hrs}$ following by abundant rinsing of sample with distilled water. After the sample was bleached, the suspension was kept in a $5 \% \mathrm{KOH}$ solution for $48 \mathrm{hrs}$ to remove any residual proteins.

(iii) Whisker Formation: In this step the micro particles are formed into nano whiskers by acid hydrolysis method.

Protein free whisker suspension was prepared by hydrolyzing the purified chitin sample with 3N HCL at the boil for $90 \mathrm{~min}$ under stirring. The ratio of $3 \mathrm{~N} \mathrm{HCL}$ to chitin was $30 \mathrm{~cm}^{3} / \mathrm{g}$. After acid hydrolysis, the suspension was diluted by transferring them to dialysis bag and dialyzed in running water for $2 \mathrm{hrs}$ and then overnight in distilled water until $\mathrm{pH} 4$ was reached. This dispersion of whisker was completed by a further $5 \mathrm{~min}$ ultrasonic treatment for every $30 \mathrm{~cm}^{3}$ aliquot. It was subsequently stored at $6^{0} \mathrm{C}$ after adding sodium azotize to avoid bacterial growth until used.

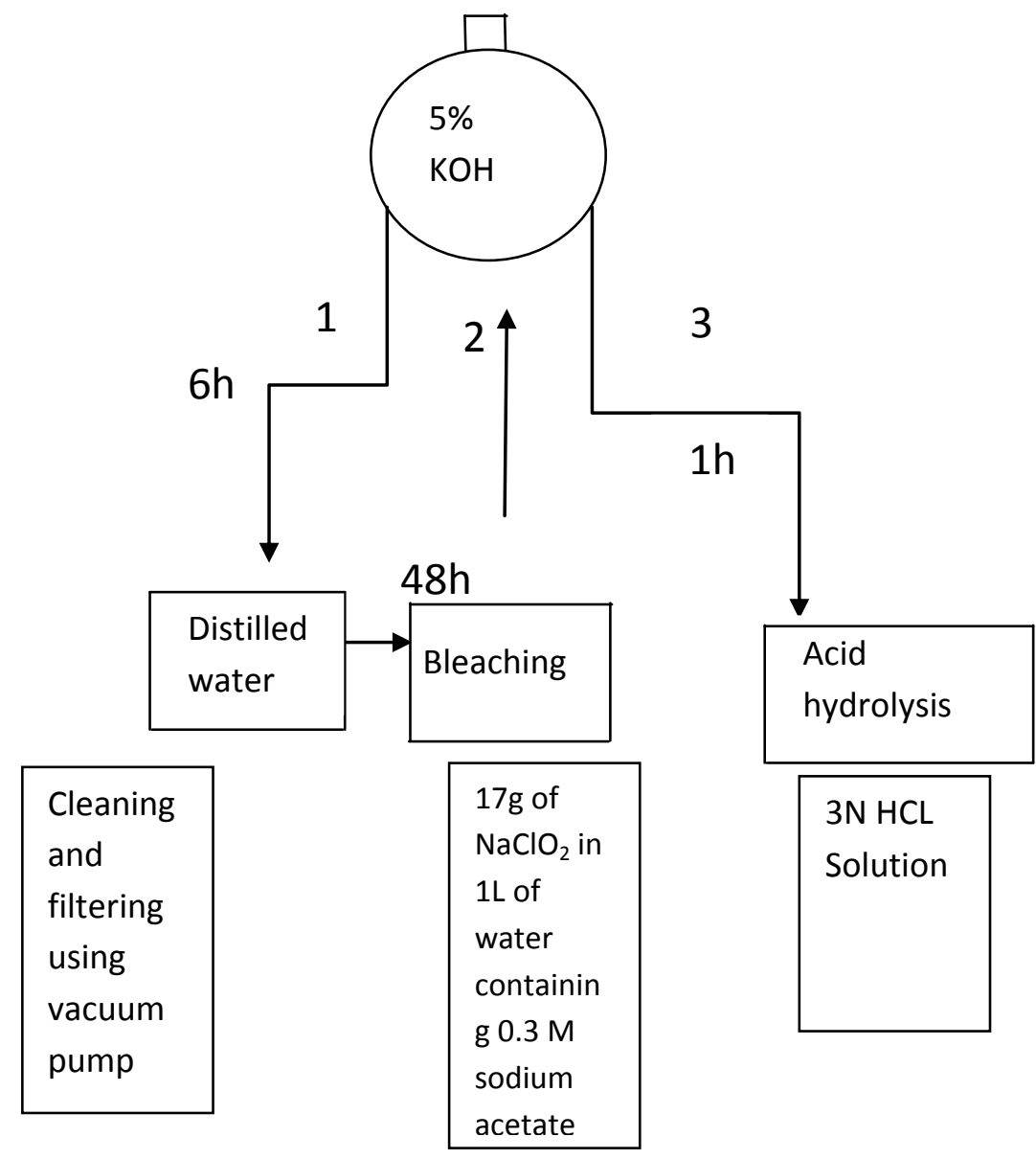

\subsection{Preparation of composites:}

Fig 1: Process of preparation of Chitin Whisker

Phenol formaldehyde sheets of certain thickness can be prepared by solvent casting process followed by hot pressing method. Water soluble Phenol formaldehyde has been used for the preparing thin sheets of thickness about $1 \mathrm{~mm}$. Phenol formaldehyde being a thermo setting polymer will cure on heating. It has been found that the water soluble phenol formaldehyde will cure at $130^{\circ} \mathrm{C}$. In the process of heating water soluble phenol formaldehyde, the phenol formaldehyde should be hot pressed so that it takes the shape of the mould. Petri dish was used to cure water soluble phenol formaldehyde in a laboratory oven

The chitin whisker suspension and water soluble phenol formaldehyde resin will be mixed in various proportions in order to obtain final dry composite films ranging between $0.5-1 \mathrm{~mm}$ in thickness with $0-20 \%$ weight fractions until the agglomeration of solid chitin whiskers in phenol formaldehyde matrix are observed and should be thoroughly stirred. These samples are casted in Petri dishes by using solvent casting method. In this the samples are heated at a temperature of $90^{\circ} \mathrm{C}$ for 24 hours(uniform distribution of heat though out the 
volume of resin) so that the water present in the medium is evaporated and then the temperature is increased up to $130^{\circ} \mathrm{C}$ to complete the chemical cross linking process.

\subsection{Characterization of Chitin Whisker:}

The chitin particles which were chemical treated with acid was converted to the nano size which was confirmed by the results obtained from DLSI technique. The figure 2 below shows the size distribution of the chitin whisker based on the intensity. The basic principle in the technique is when laser light hits small particles suspended in a liquid, it scatters in all directions and one may observe time dependent intensity fluctuations in the scattering caused by the particle interaction with the surrounding solvent molecules. The intensity fluctuations are caused by motion of the particles and solvent which produce constructive and destructive interference of the light which gives the information about the hydrodynamic size of the particles lies therein. Morphology of Chitin Particles was also analyzed by the SEM images for its size confirmation.

\section{Results}

The figure 1 shows the DLSI results indicating the scattered light intensity for various sized particle showing the highest peak intensity for the particle with a size from 100 to $5000 \mathrm{~nm}$ the results have been presented in the form of table below concentrating on the highest peak intensity. The length and width dimensions of the nano particles can be observed by the DLSI technique which gives a histogram obtained by taking the size of the particle along its length on its abscissa and the percentage intensity of the particles on the ordinate. The nano particles suspension obtained from the acid hydrolysis process when tested by DLSI technique the result was as given in the figure (2).

The length of the particles were found to be ranging from 150 to $5000 \mathrm{~nm}$ and there width ranging from 80 to $450 \mathrm{~nm}$. More than $60 \%$ of the whiskers have a length below $200 \mathrm{~nm}$ length and $80 \mathrm{~m}$ width. The average length of particle is $220 \mathrm{~nm}$ and the width was estimated to be around $80 \mathrm{~nm}$. The average aspect ratio (L/d, were $\mathrm{L}$ and $\mathrm{d}$ are the length and width of the particle) was found to be 2.75 approximately.

\section{Dynamic Light Scattering Imaging}

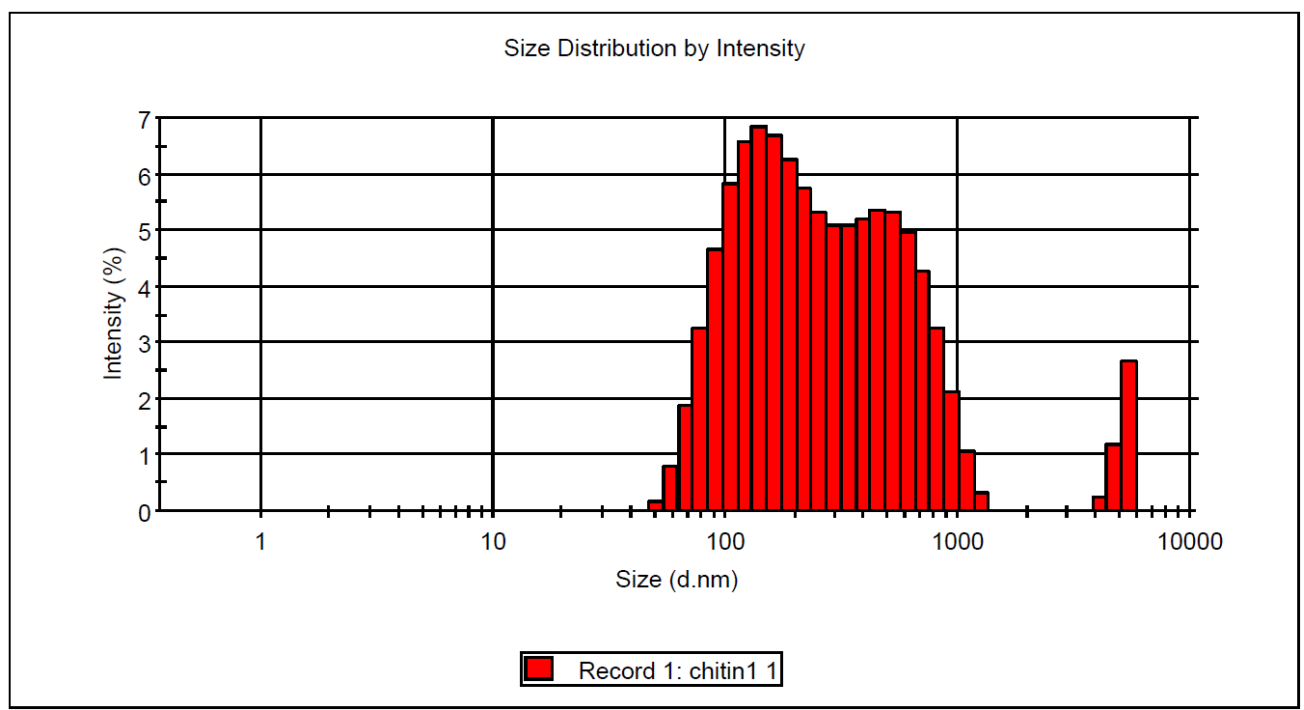

\section{SYSTEM}

Temperature $\left({ }^{\circ} \mathrm{C}\right): 25.0$

Duration Used(s): 60

Count rate (KCPS):207.9

Measurement Position (mm): 4.65

Cell description: Disposable Sizing Cuvette

Attenuator: 6

Fig 2: Size Distribution by Intensity

$\begin{array}{rllll}\text { Z-Average(d.nm): } 220.9 & \text { Peak1: } & 177.8 & 61.0 & 80.33 \\ \text { Pdl: } 0.638 & \text { Peak2: } & 580.4 & 35.1 & 206.1 \\ \text { Intercept: } 0.938 & \text { Peak3: } & 5267 & 3.9 & 431.3\end{array}$

Table 1: Size Distribution by Intensity 
The figure 2 shows a SEM of a dilute suspension of shell chitin which consists of chitin nano particles. These particles are seen to be having the shape as mentioned by Mr. K.G.Nair (4) as slender rods with sharpen points that has a broad distribution in size. The length and width dimension of the nano particles are satisfactory when compared with the DLSI results.

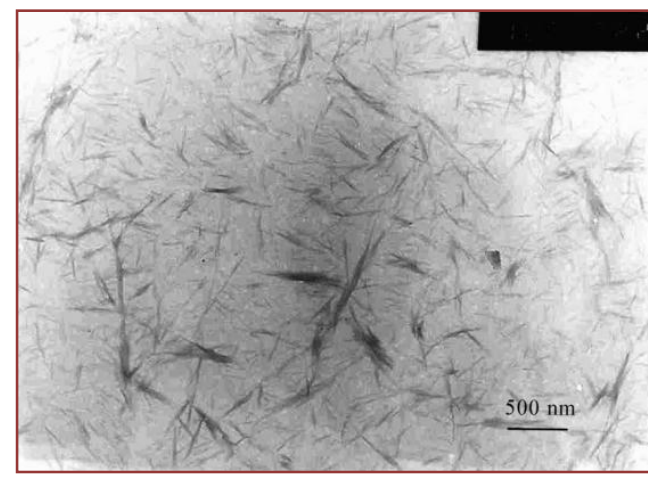

Fig 3: Morphology of Chitin Nano Particles suspension

\section{Conclusion:}

Chitin whiskers colloidal suspension was prepared for development of chitin reinforces phenol formaldehyde composites. The chitin whiskers, prepared by acid hydrolysis technique have shape of parallelepiped slender rod with an average length of $220 \mathrm{~nm}$ and an aspect ratio close to 2.75 . Which when compares with the SEM image gives the confirmation of the same. The size of the particles obtained by acid hydrolysis method was satisfactory when compared to the results from the literature. The polymer particles exhibiting ultrafine phase dimension that fall in the range of 1 to $1000 \mathrm{~nm}$ which may have some unique outstanding properties with respect to their conventional micro composite counterparts $(6,7)$ which showed an enhanced reinforcing thermal and sorption properties compared to the pure phenol formaldehyde material on curing.

\section{References}

[1]. Xiao-ming Tan, Hong-quan xie and Nai-Yu Huang, (2002), Preparation and photosynthasis of water soluble phenolic resin containing acryloyl and quarternary ammonium chloride group, Chinese Journal of Polumer Scienece Vol. 20. No. 2, pp. 129-136.

[2]. Kalaprasad Gopalan Nair and Alain Dufresne, (2003), Crab Shell Chitin Whisker Reinforced Natural Rubber Nanocomposites. 1. Processing and Swelling Behavior, Biomacromolecules ,No. 4, pp. 657-665.

[3]. Ahmed Said Azizi Samir,Fannie Alloin and Alain Dufresne, (2005), Review of Recent Research into cellulosic Whiskers, Their Properties and Their Application in Nanocomposite Field, Biomacromolecules, No. 6, pp. 612-626.

[4]. Arnaud morin and Alain Dufrene, (2002), Nano Composites of Chitin Whisker from Riftia Tubes and Poly(, Macromolecules, Vol. No. 35,pp. 2190-2199.

[5]. R.S.Umamaheshwar Rao and Dr. G.Chandra Mohan Reddy, (2011), Development And Tharmal Characterization Of Chitin Particle Reinforced Phenol Formaldehyde Composite, Institute of Engineerins Journal-CH, Vol. No. 92, pp. 8-11.

[6]. R.S.Umamaheshwar Rao and Dr. G.Chandra Mohan Reddy, 2009, Preparation And Characterization Of Chitin Reinforced Phenol Formaldehyde Composite, International Conference on Computational Methods in Engg. \& Sciences .

\section{Figure Captions}

Fig 1: Process of preparation of Chitin Whisker

Fig 2: Size Distribution by Intensity

Fig 3: Morphology of Chitin Nano Particles suspension

\section{List of Tables}

\section{Table 1: Size Distribution by Intensity}

\section{List of Symbols}

d Diameter

SEM Scanning electron microscope

DLSI Differential scanning calorimeter

HCL Hydrochloric acid

$\mathrm{KOH} \quad$ Potassium hydroxide

$\mathrm{NAClO}_{2} \quad$ Sodium hypochlorite 